\title{
Diferencias de género en el consumo diario de tabaco e intensivo de alcohol en adolescentes latinoamericanos en tres áreas españolas (Andalucía, Madrid y País Vasco).
}

\author{
Gender differences to daily smoking and binge drinking in the Latinoamerican Adoles- \\ cents in three Spanish areas.
}

\begin{abstract}
Carmen Meneses ${ }^{\text {a }}$, Iñaki Markez ${ }^{\mathrm{b}}$, Nuria Romo ${ }^{\mathrm{c}}$, Jorge Uroz $^{\mathrm{d}}$, Antonio Rua ${ }^{\mathrm{e}}$, Teresa Laespada ${ }^{\mathrm{f}}$.
${ }^{a}$ Dra. en Antropología. Universidad Pontificia Comillas, Madrid, España. ${ }^{b}$ Dr. en Medicina y Cirugía, Psiquiatra. Ekimen2000, Bilbao, España. ${ }^{c}$ Dra. en Antropología. Universidad de Granada, Granada, España. ${ }^{d}$ Dr. en Sociología. Universidad Pontificia Comillas, Madrid, España. ${ }^{e}$ Dr. en Estadística. Universidad Pontificia Comillas, Madrid, España ${ }^{f}$ Dra. en Sociología. Universidad de Deusto, Bilbao, España.
\end{abstract}

Correspondencia: Carmen Meneses Falcón (cmeneses@chs.upcomillas.es)

Recibido: 23/09/2012; aceptado: 18/01/2013

RESUMEN: El consumo diario de tabaco e intensivo de alcohol en la adolescencia puede configurarse como hábitos persistentes para la vida adulta. En este trabajo se pretende explorar las diferencias entre chicos y chicas adolescentes de origen latino con consumos intensivos de tabaco y alcohol, dado que son escasos los trabajos en España que introducen la variable cultural o étnica. Se trata de un estudio trasversal mediante encuesta escolar, a 1126 adolescentes de origen latino, escolarizados en la Educación Secundaria en tres zonas españolas, de 12 a 19 años. Entre los resultados destacamos que el $9,4 \%$ de los chicos y el $5,6 \%$ de las chicas latinas fumaban diariamente y el $24,2 \%$ de los chicos y el $19 \%$ de las chicas bebió cinco o más bebidas alcohólicas en el último evento. Fumar diariamente se asoció con ser chico, fumar el padre, fumar la pareja, actuando como factor de protección hacer deporte. Ser chico, emborracharse y haber ido de botellón aumenta la probabilidad de consumir alcohol de forma intensiva. Para concluir destacamos que existen diferencias de género en los consumos de tabaco $\mathrm{y}$ alcohol en el tipo de consumo intensivo pero no se aprecia cuando se trata del uso de estas sustancias. Se plantea la necesidad de tener en cuenta la variable de origen cultural en las intervenciones educativas sociosanitarias.

PALABRAS CLAVE: salud del adolescente, inmigrantes, consumo de bebidas alcohólicas, tabaco, identidad de género.
ABSTRACT: The daily smoking and binge drinking in adolescence may produce persistent habit for adulthood. The objective of this research is to explore the differences between Latinoamerican girls and boys adolescents in the tobacco and alcohol consumes. The researches with cultural variable are scant in Spain. This study is transversal via a school survey comprising 1126 Latinoamerican adolescents, in Secondary Education in three areas and ranging from 12 to 19 years of age. The results are the $9,4 \%$ of the boys and the $5,6 \%$ of the girls smoke daily, and the $24,2 \%$ of the boys and the $19 \%$ of the girls for binge drinking the last event. The probability of daily smoking was associated to be a boy, to have a smoking father, to have a smoking couple and as protection factor was to do sport. So, to be a boy, to get drunk and have gone to Botellon increase the probability to binge drinking. The most important conclusion is that there were differences of gender in tobacco and alcohol abuse but not in the use. It is necessary to introduce the cultural variable in educative, health and social prevention.

KEY WORDS: Adolescent Health, immigrants, alcohol drinking, tobacco, gender identity.

\section{Agradecimientos:}

Los autores agradecen a todos los centros educativos de las tres Comunidades Autónomas la colaboración en este estudio, a los estudiantes y a sus padres que permitieron también su realización. Este trabajo forma parte del Proyecto Nacional de I+D+I, "Riesgo, Adolescencia y Etnicidad: comparando tres áreas geográficas de España”, subvencionado por el Ministerio de Ciencia e Innovación, Referencia: CSO2009-07732. 


\section{Introducción}

En el año 2009, se contabilizó casi cuatro millones ochocientas mil personas extranjeras residiendo en España, de las cuales el 30\% procedían de Iberoamérica, segunda zona más importante de origen de los inmigrantes en el país, y casi un $15 \%$ de esta población tenía entre 10 y 19 años, más de doscientos mil adolescentes de este intervalo de edad según la Secretaria General de Inmigración y Emigración (1). Aunque son muchos los estudios realizados en España en las últimas décadas sobre la población inmigrante pocos se han centrado en los adolescentes y sus hábitos de salud. Se ha planteado que la etapa de la adolescencia es un momento de formación, experimentación y aprendizaje en diferentes conductas que marca o sienta las bases para los hábitos en la vida adulta (2). Se ha señalado que la etapa adolescente no es homogénea ni universal pues, a pesar de vivir en la era de la globalización la diversidad cultural imprime notables diferencias (3). En este sentido la pertenencia a un grupo étnico, o la etnicidad, es un elemento muy importante de identificación social y cultural que modela a los adolescentes en sus pautas de comportamiento, y conforma su identidad en el transito a la vida adulta. En la sociedad española conviven diferentes grupos étnicos y los procesos de interacción intercultural, especialmente entre los adolescentes, pueden causar cambios en sus comportamientos de salud. El consumo de tabaco y alcohol son dos acontecimientos que emergen en la adolescencia y aunque son los adultos los que más consumen estas sustancias, cuando lo hacen los adolescentes suelen hacerlo con mayor intensidad y frecuencia en un breve periodo de tiempo. Los consumos de drogas varían por género y etnicidad (4-6) pues se ha señalado que las chicas caucasianas fuman y beben en mayor medida que las chicas de otras minorías étnicas (7). Se ha planteado que los adolescentes latinos, no asimilados a la cultura mayoritaria, presentan menores consumos de drogas que aquellos que se asimilaron o integraron en la sociedad receptora (8). También se ha mencionado que los adolescentes latinos o de origen hispano tienen menores consumos de alcohol, sobre todo en la primera generación, que los adolescentes blancos-caucasianos (8), con diferentes factores de riesgo y protección en cada uno de ellos (9). Pertenecer a un grupo étnico minoritario se ha planteado como un factor de protección para el consumo diario de tabaco o alcohol (10). Por otra parte, las diferencias de género se unen a las étnicas, siendo los chicos los que muestran mayores consumos intensivos de alcohol y tabaco $(7,9)$ y las chicas latinas tienen una menor frecuencia de consumo de alcohol con forme avanza la edad (11). Estudios realizados en el contexto europeo sobre población adolescente, autóctona y no autóctona, han mostrado como las normas familiares en los consumos de alcohol influyen en el consumo de los adolescentes (12). Por otra parte se ha planteado que la prevención del consumo de drogas no es igual de efectivo cuando existe una heterogeneidad respecto al sexo y a la etnia (9). 
ORIGINALES Y REVISIONES

La amplia literatura sobre consumo y abuso de drogas ha mencionado muy diversos factores de riesgo para el consumo de sustancias psicoactivas, sin embargo, se asume que estos factores son los mismos para el uso, el abuso o la dependencia a las drogas (13).

El objetivo de este trabajo es explorar las diferencias intersexuales en el consumo diario de tabaco e intensivo de alcohol entre adolescentes de origen latinoamericano establecidos en España, que nos permitan proponer líneas de intervención preventiva del consumo de estas drogas, ya que suponen una de las mayores causas de muerte prematura en población adulta.

\section{Métodos}

\section{Diseño del estudio}

Estudio trasversal mediante encuesta escolar recogida, durante los meses de enero a julio del 2011, a estudiantes de Secundaria Obligatoria en centros educativos con mayor concentración de adolescentes de origen latinoamericano, en Andalucía, Madrid y País Vasco. La encuesta fue autorizada por los centros educativos seleccionados.

\section{Participantes y recolección de datos}

Inicialmente se planteo un muestreo aleatorio simple como diseño muestral. Partiendo de datos del Ministerio de Educación ${ }^{1}$ en España en el curso 2009-2010, se estimó que el número de alumnos latinos matriculados en el ESO en Andalucía ascendía a 7.972, en Madrid a 20.377 y en el País Vasco a 3.026, lo que implica una población final de latinos matriculados en la ESO en estas tres comunidades de 31.375. Proyectando la estimación de ese tamaño poblacional de alumnos latinos, y considerando un tamaño muestral de 1000 alumnos, implica, con estimación de los tamaños muestrales para $\mathrm{p}=\mathrm{q}=0,5$, un error muestral de un $3 \%$ con un grado de confianza del $95 \%$. Si se realizase un muestreo proporcional al tamaño de cada comunidad, afijación proporcional, la cuotas correspondientes a cada comunidad serían: en Andalucía un n=254, en Madrid un n= 650 y en el País Vasco un n=96. Este tamaño se tuvo como referencia mínima para conseguir la muestra. La muestra final conseguida fue de 128 en Andalucía, pues no se consiguió llegar a la cuota establecida, 748 en Madrid y 250 en el País Vasco, superando en ambos casos lo planeado, formando una muestra total de 1126 adolescentes latinos, lo que de forma global para las tres comunidades reduce el error muestral a un $2,9 \%$, con un grado de confianza del $95 \%$.

\footnotetext{
1 (http://www.educacion.es/mecd/jsp/plantilla.jsp?id=31\&area=estadisticas)
} 
Para recoger el mayor número de cuestionarios contactamos con los directores y orientadores educativos de los centros en cada Comunidad Autónoma y nos confirmaron el alto porcentaje de estudiantes matriculados de origen latino. El cuestionario lo cumplimentaron todos los alumnos de las aulas seleccionadas y posteriormente el equipo seleccionó para la muestra aquellos alumnos que se autoidentificaron como latinoamericanos. No obstante además de la autoidentificación de los propios adolescentes, se recogió el lugar de nacimiento de ellos y de sus padres. El instrumento de recogida de información fue un cuestionario confidencial y autocumplimentado, que fue validado en una investigación anterior (14). No obstante, se realizaron algunas modificaciones al mismo y antes de utilizarlo se efectuaron dos grupos de discusión con adolescentes y una prueba pre-test en un centro educativo no incluido en la muestra que sirvieron para valorar dichas modificaciones. Los padres fueron informados de la investigación por los centros educativos y éstos se encargaron de recabar la autorización. Los datos fueron recogidos en las aulas por los propios investigadores ayudados por encuestadores. La cumplimentación del cuestionario se realizaba en 40 o 45 minutos.

\section{Variables de estudio}

El cuestionario recogía diferentes comportamientos de riesgo, pero en este artículo solo expondremos los relacionados con el consumo de tabaco y alcohol. Las variables de estudio fueron recogidas para el último año, salvo en el caso del tabaco que se les preguntó también si fumaban en ese momento. Se obtuvo un factor que evaluaba las creencias religiosas a través de seis variables recogidas (Mi religión es muy importante para mí, Me considero una persona muy creyente, Mis creencias religiosas influyen en mis acciones, Cumplo con los mandatos de mi religión, Rezo todos los días, Disfruto estando con otras personas tan religiosas como yo) con cuatro posibilidades de respuestas (Muy de acuerdo, de acuerdo, en desacuerdo y muy en desacuerdo), con alfa de Cronbach de 0,906 (tanto en esta muestra como en muestras realizadas con anterioridad en población adolescente). Las variables dependientes fueron dicotomizadas para los análisis. Se recogió el número de bebidas alcohólicas (vasos, copas, bebidas) en el último evento y posteriormente se realizaron dos intervalos: menos de 5 copas y 5 o más copas. Se recogió la frecuencia de consumo de tabaco (Nunca, algunas veces al año, dos o tres veces en semana, fines de semana y a diario) y desde esta variable se construyo una variable dicotómica (fuma diariamente o no fuma diariamente).

Análisis de datos

Con el fin de probar la independencia de las variables se utilizó el estadístico chi cuadrado y t-student dependiendo del tipo de variable y el supuesto a contrastar. El modelo de regresión logística fue construido separadamente para cada comportamiento de consumo estudiado (fumar diariamente y consumo intensivo de alcohol). La prevalencia de odds ratios (OR) fue calculada con un nivel de confianza 
ORIGINALES Y REVISIONES

del $95 \%$ con sus intervalos de confianza correspondientes. Para todos los análisis se calculo la significación estadística en el 5\%. Los análisis fueron realizados con el paquete informático SPSS v.17.

\section{Resultados}

Datos sociodemográficos. El 48\% fueron chicos, la edad media fue de 15,18 años (DS=1,57), en un intervalo de 12 a 19 años. El 18,7\% percibían que iba bien económicamente en sus casas, el 61,5\% señaló no tener problemas económicos y el $19,9 \%$ muchos problemas económicos. El número medio de personas que convivía en el hogar era de 4 personas ( $\mathrm{DS}=1,55)$. El 48,5\% había repetido algún curso durante la ESO. El 63,5\% vivía habitualmente con su padre y el 92,3\% con su madre.

Diferencias entre chicos y chicas. En la tabla 1 se muestra el consumo de estas sustancias en toda la muestra y entre sexos, encontrando diferencias significativas en algunas variables. Fumar diariamente era significativo entre sexos, siendo mayor en los chicos, pero no era así en el caso del consumo intensivo de alcohol, que no se encontraron diferencias.

Fumar diariamente y consumo intensivo de alcohol. En la tabla 2 se muestra los resultados obtenidos del análisis bivariado, segmentado por sexo, donde se puede apreciar que salvo en algunas variables todas resultan significativas y se alcanzan mayores porcentajes entre los que fuman diariamente y beben alcohol intensivamente. En la tabla 3 se muestra los principales resultados de regresión logística. Ser chico, fumar el padre y que fume la pareja aparecen como factores de riesgo para fumar diariamente, mientras que hacer deporte surge como factor de protección; Ser chico, ir de botellón a beber y emborracharse se mostraron como factores de riesgo para el consumo intensivo de alcohol. El factor religioso podría interpretarse en ambos comportamientos como protección hacia estos comportamientos. 
Tabla 1.

Consumo de tabaco y alcohol en los últimos 12 meses y comportamientos de riesgo asociados por sexo. $n(\%)$.

\begin{tabular}{|c|c|c|c|c|}
\hline & $\begin{array}{l}\text { Total } \\
\mathrm{N}(\%)\end{array}$ & $\begin{array}{l}\text { CHICOS } \\
\mathrm{N}(\%)\end{array}$ & $\begin{array}{l}\text { CHICAS } \\
\mathrm{N}(\%)\end{array}$ & $\mathrm{p}$ \\
\hline Fuma en el momento encuesta (si) & $162(14,8)$ & $86(16,4)$ & $76(13,3)$ & 0,145 \\
\hline $\mathrm{N}^{\circ}$ cigarrillo (X y DS)a & $2,86(3,59)$ & $3,19(3,70)$ & $2,46(3,46)$ & 0,169 \\
\hline Diario (si) & $80(7,4)$ & $48(9,4)$ & $32(5,6)$ & 0,019 \\
\hline Fuma los padres (si) & $312(28,7)$ & $157(30,1)$ & $155(27,4)$ & 0,317 \\
\hline Fuma el padre & $227(20,2)$ & $117(21,7)$ & $110(18,9)$ & 0,231 \\
\hline Fuma la madre & $131(11,7)$ & $60(11,2)$ & $71(12,2)$ & 0,593 \\
\hline Fuma la pareja & $107(18,9)$ & $37(12,9)$ & $70(25,2)$ & 0,000 \\
\hline Fuma la mayoría de los amigos & $808(74,5)$ & $381(73,4)$ & $427(75,6)$ & 0,414 \\
\hline Alcohol (si) & $605(62,0)$ & $287(61,2)$ & $318(62,7)$ & 0,623 \\
\hline \multicolumn{5}{|l|}{ Contexto consumo alcohol } \\
\hline Con la familia & $294(26,2)$ & $123(22,9)$ & $171(29,3)$ & 0,014 \\
\hline Fuera de la familia & $523(46,7)$ & $242(45,0)$ & $281(48,2)$ & 0,281 \\
\hline Beber solo varias veces (SI) & $95(9,1)$ & $47(9,5)$ & $48(8,8)$ & 0,708 \\
\hline $\mathrm{N}^{\circ}$ de copas último evento $(\mathrm{X} \text { y } \mathrm{DS})^{\circ}$ & $2,82(4,21)$ & $3,06(5,05)$ & $2,59(3,24)$ & 0,104 \\
\hline Ha bebido en Botellón (SI) & $521(56,7)$ & $252(55,4)$ & $269(58,0)$ & 0,428 \\
\hline 5 o más bebidas último evento (SI) & $190(21,5)$ & $103(24,2)$ & $87(19,0)$ & 0,061 \\
\hline Emborracharse (SI) & $434(40,2)$ & $201(39,3)$ & $233(41,1)$ & 0,522 \\
\hline Hace deporte (SI) & $832(74,4)$ & $487(90,5)$ & $345(59,5)$ & 0,000 \\
\hline Factor religioso $(\mathrm{X} \text { y DS })^{\circ}$ & $-0,124(0,89)$ & $-0,193(0,90)$ & $-0,057(0,88)$ & 0,017 \\
\hline Hacer botellón y montar en moto después (Nunca) & $852(78,7)$ & $395(77,0)$ & $457(80,3)$ & 0,244 \\
\hline $\begin{array}{l}\text { Tener relaciones sexuales estando muy bebido o } \\
\text { consumido alguna droga (Nunca) }\end{array}$ & $902(84,5)$ & $408(79,5)$ & $494(89,0)$ & 0,000 \\
\hline Pelarme con otros habiendo bebido (Nunca) & $973(90,1)$ & $436(85,0)$ & $537(94,7)$ & 0,000 \\
\hline Tomar medicamentos con alcohol (Nunca) & $1020(94,6)$ & $480(93,8)$ & $540(95,4)$ & 0,053 \\
\hline Emborracharse y perder el sentido (Nunca) & $939(87,1)$ & $448(87,3)$ & $491(86,9)$ & 0,062 \\
\hline $\begin{array}{l}\text { Emborracharse todos los fines de semana del } \\
\text { último mes (Nunca) }\end{array}$ & $962(89,1)$ & $449(87,5)$ & $513(90,5)$ & 0,283 \\
\hline
\end{tabular}

${ }^{a} X$ (Media); DS (Desviación estándar) 
Tabla 2.

Factores asociados al consumo diario de tabaco y consumo intensivo de alcohol.

\begin{tabular}{|c|c|c|c|c|}
\hline & $\begin{array}{c}\text { CHICOS } \\
\text { Fuma Diario }\end{array}$ & $\mathrm{p}$ & $\begin{array}{c}\text { CHICAS } \\
\text { Fuma Diario }\end{array}$ & $\mathrm{p}$ \\
\hline & $\mathrm{N}(\%)$ & & $\mathrm{N}(\%)$ & \\
\hline Edad (X y DS) & $15,5(1,51)$ & 0,084 & $16,1(1,44)$ & 0,000 \\
\hline $\mathrm{N}^{\circ}$ cigarrillo (X y DS)a & $5,51(3,75)$ & 0,000 & $4,95(3,69)$ & 0,000 \\
\hline Fuma los padres (si) & $31(64,6)$ & 0,000 & $13(43,3)$ & 0,000 \\
\hline Fuma el padre & $24(50)$ & 0,000 & $8(25,9)$ & 0,400 \\
\hline Fuma la madre & $12(25)$ & 0,00 & $12(37,5)$ & 0,000 \\
\hline Fuma la Pareja (sí) & $11(39,3)$ & 0,000 & $8(50,0)$ & 0,017 \\
\hline Fuma la mayoría de Amigos & $47(100)$ & 0,000 & $30(96,8)$ & 0,005 \\
\hline Factor religioso(X y DS)a & $-0,49(0,81)$ & 0,017 & $-0,59(0,87)$ & 0,001 \\
\hline \multirow[t]{2}{*}{ Hace deporte (Sí) } & $41(85,4)$ & 0,213 & $14(43,8)$ & 0,049 \\
\hline & $\begin{array}{c}5 \text { o más } \\
\text { Bebidas } \\
\mathrm{N}(\%)\end{array}$ & $\mathrm{p}$ & $\begin{array}{c}5 \text { o más } \\
\text { Bebidas } \\
\mathrm{N}(\%)\end{array}$ & $\mathrm{p}$ \\
\hline \multicolumn{5}{|l|}{ Consumo alcohol } \\
\hline Con la familia & $34(33,0)$ & 0,016 & $38(43,7)$ & 0,002 \\
\hline Fuera de la familia & $81(78,6)$ & 0,000 & $71(81,6)$ & 0,000 \\
\hline Beber solo varias veces (si) & $15(14,6$ & 0,002 & $11(12,6)$ & 0,137 \\
\hline $\mathrm{N}^{\circ}$ de copas último evento (X y DS) & $9,21(7,05)$ & 0,000 & $8,02(3,19)$ & 0,000 \\
\hline Ha bebido en Botellón (sí) & $89(90,8)$ & 0,000 & $75(93,8)$ & 0,000 \\
\hline Se ha emborrachado & $78(77,2)$ & 0,000 & $67(77,9)$ & 0,000 \\
\hline Factor religioso (X y DS)a & $-0,30(0,83)$ & 0,168 & $-0,30(0,86)$ & 0,046 \\
\hline Hace deporte (Sí) & $96(93,2)$ & 0,340 & $49(56,3)$ & 0,338 \\
\hline Hacer botellón y montar en moto después (Nunca) & $61(60,4)$ & 0,000 & $59(67,8)$ & 0,000 \\
\hline $\begin{array}{l}\text { Tener relaciones sexuales estando muy bebido o } \\
\text { consumido alguna droga (Nunca) }\end{array}$ & $52(52,0)$ & 0,000 & $61(72,6)$ & 0,000 \\
\hline Pelarme con otros habiendo bebido (Nunca) & $66(65,3)$ & 0,000 & $72(83,7)$ & 0,000 \\
\hline Tomar medicamentos con alcohol (Nunca) & $92(92,0)$ & 0,125 & $77(89,5)$ & 0,002 \\
\hline Emborracharse y perder el sentido (Nunca) & $70(69,3)$ & 0,000 & $51(60,7)$ & 0,000 \\
\hline $\begin{array}{l}\text { Emborracharse todos los fines de semana del } \\
\text { último mes (Nunca) }\end{array}$ & $72(72,0)$ & 0,000 & $64(74,4)$ & 0,000 \\
\hline
\end{tabular}

${ }^{a} X$ (Media); DS (Desviación estándar)

Tabla 3.

Factores asociados al consumo diario de tabaco y consumo intensivo de alcohol.

\begin{tabular}{|l|c|c|c|c|c|}
\hline Fumar diariamente $^{\mathbf{a}}$ & $\mathbf{B}$ & WALD & $\mathbf{p}$ & OR & (IC95\%) \\
\hline Fuma el padre & 0,971 & 6,344 & 0,012 & 2,64 & $1,24-5,62$ \\
Fuma la madre & 0,770 & 3,197 & 0,074 & 2,15 & $0,92-5,02$ \\
Fuma la pareja & 1,366 & 11,702 & 0,001 & 3,92 & $1,79-8,57$ \\
Hace deporte & $-1,470$ & 10,540 & 0,001 & 0,23 & $0,09-0,55$ \\
Factor religioso & $-0,177$ & 0,741 & 0,389 & 0,83 & $0,55-1,25$ \\
Sexo & 1,602 & 11,956 & 0,001 & 4,96 & $2,00-12,29$ \\
\hline Cinco o más bebidas alcohólicas & & & & & \\
\hline Bebe con la familia & 0,117 & 0,282 & 0,595 & 1,12 & $0,72-1,73$ \\
Bebe fuera de la familia & 0,159 & 0,341 & 0,559 & 1,17 & $0,68-1,99$ \\
Ha ido de botellón a beber & 1,963 & 29,365 & 0,000 & 7,11 & $3,50-14,47$ \\
Se ha emborrachado & 1,173 & 23,044 & 0,000 & 3,23 & $2,00-5,21$ \\
Bebe solo varias veces & 0,234 & 0,568 & 0,451 & 1,26 & $0,68-2,32$ \\
Hace deporte & 0,226 & 0,696 & 0,404 & 1,25 & $0,73-2,13$ \\
Factor religioso & $-0,069$ & 0,317 & 0,574 & 0,93 & $0,73-1,18$ \\
Sexo & 0,577 & 6,471 & 0,011 & 1,78 & $1,14-2,77$ \\
\hline
\end{tabular}

${ }^{a} R 2$ de Cos y Snell=0,080; $R 2$ de Nagelkerke $=0,189 ;{ }^{b} R 2$ de Cos y Snell=0, 218; 22 de Nagelkerke $=0,328$ 
ORIGINALES Y REVISIONES

\section{Discusión}

Este estudio pone de relieve algunos factores de riesgo para el consumo intensivo de tabaco y alcohol en adolescentes de origen latino establecidos en España, con claras diferencias de género, ya que eran los chicos los que mostraron un mayor riesgo para este tipo de consumo. La convergencia del consumo de drogas legales entre chicos y chicas adolescentes que se muestra en las encuestas escolares no parece hallarse en la muestra de adolescentes estudiada. Los resultados obtenidos confirman que los comportamientos de consumo de drogas, especialmente los intensivos, varían significativamente por género dentro de los grupos étnicos, tal como se ha mencionado en otros trabajos $(8,15,16)$.

Si comparamos los datos obtenidos en los adolescentes latinos con los datos de la encuesta nacional del Observatorio Español sobre Drogas, ESTUDES, 2009 (17), los adolescentes latinos muestran menores prevalencias de consumo diario de tabaco, comparado con los adolescentes autóctonos (7,4\%-15\% respectivamente). Mientras que la encuesta nacional marca diferencias entre chicos y chicas, siendo éstas últimas las que alcanzan mayores porcentajes de consumo de tabaco diariamente, en la muestra de latinos estudiada en este trabajo ocurre lo contrario, eran los chicos los mayores fumadores diarios. En el consumo intensivo de alcohol, valorado como en otros estudios en más de cinco bebidas alcohólicas en el último evento, encontramos la misma situación. Mientras que en la encuesta nacional el $41,4 \%$ ha tenido esta conducta, en la muestra de latinos analizada era alrededor de la mitad $(21,5 \%)$. Sin embargo, aunque sigue siendo algo mayor en la encuesta nacional, las borracheras en el último año no muestran grandes diferencias porcentuales $(47,1 \%$ en encuesta nacional y $40,2 \%$ en latinos) ni por sexo en los datos nacionales respecto a la muestra de latinos analizada. Estas diferencias encontradas pueden ser explicadas por los procesos de aculturación. El alcohol es un producto de consumo social, entre el grupo de iguales y que produce una experiencia de placer y comunión con el grupo (18), que les ofrece a los adolescentes una oportunidad de integración. En este sentido más de la mitad de la muestra ha acudido a un Botellón a beber, sin diferencias intersexuales. Es posible que estos usos de drogas, especialmente los intensivos y de riesgo, estén relacionados con los procesos de aculturación y de configuración de la identidad, pues uno de los factores de riesgo más importante asociados a estas conductas de riesgo es que el grupo de iguales las haga. En este sentido otros trabajos han puesto de relieve como las conductas de consumo del grupo de iguales pueden ser un predictor para el consumo de los adolescentes (19-20).

Se han hallado diferencias de sexo en ciertos comportamientos de riesgo asociado con el consumo de alcohol. Cuando el consumo de alcohol era incorporado con otras conductas diferentes como la conducción, o mantener relaciones sexua- 
les, las chicas de origen latino se implicaban menos. Sin embargo, no había diferencias entre chicos y chicas en aquellas conductas de riesgo que solo suponía el consumo de alcohol.

Nos falta evidencia en la manera en que los padres influyen en el consumo de tabaco y alcohol de sus hijos (21-22) y cómo esto varía dependiendo de la variable cultural, pero los datos obtenidos en este trabajo en adolescentes latinos nos muestran que el que el padre fume es un factor de riesgo para fumar diariamente su hijo. Que la influencia del padre sea mayor que la de la madre puede estar relacionada con los modelos de consumo masculinos. Generalmente la iniciación en el consumo de tabaco suele realizarse entre iguales, pero es posible que para la adquisición del tabaquismo los modelos familiares sean más influyentes. No sucede lo mismo con el consumo de alcohol, pues no resulta un factor de riesgo su consumo en el contexto familiar. Esto es posible porque el consumo que se realice en el ámbito familiar esté circunscrito a celebraciones o ritualizado en costumbres diarias, siendo diferente al que realizan entre iguales. No obstante, se requiere un estudio más amplio y no trasversal para estudiar la dinámica de relación con los padres y el grupo de iguales en el consumo de drogas, especialmente para la transición del consumo experimental u ocasional al consumo intensivo de tabaco y alcohol. La familia probablemente sea un segundo contexto de consumo, aunque los roles jugados en la iniciación son complejos y se necesita una mayor profundización.

El riesgo y las conductas de riesgo han sido valorados desde diferentes enfoques y perspectivas $(3,23)$. Por una parte se han planteado que muchas conductas de riesgo son una forma de experimentación, aprendizaje y control de límites que precisan los adolescentes para su crecimiento y maduración personal. Por otro lado, se ha planteado que estas conductas de riesgo, relacionadas con distintos factores que las pueden propiciar, dejan un saldo negativo importante para los propios adolescentes en términos de accidentalidad y mortalidad, dejando secuelas en su desarrollo. Podemos valorar de forma diferente los dos comportamientos de riesgo estudiados en este trabajo. Es posible que las conductas relacionadas con el alcohol tengan un componente muy importante social, más que individual o familiar, y puedan ser valoradas desde el primer planteamiento expuesto. Desde esta perspectiva, articular las medidas de disminución de riesgo sea más eficaz con adolescentes latinos y autóctonos, pues conjuntamente se producen muchos de sus consumos. La perspectiva de intervención debe ser preferentemente social, lo individual puede quedar relegado a un segundo plano. En cambio el consumo intensivo o diario de tabaco, aunque el grupo juegue un papel importante en la iniciación, es una conducta más individual, pues se fuma en solitario, y quizás los mensajes preventivos así como las intervenciones pueden realizarse en este plano.

Debemos señalar algunas limitaciones de este trabajo, principalmente relacionadas con la muestra. Aunque dicha muestra es amplia y diversa, no es represen- 
tativa de los adolescentes de origen latinoamericano establecidos en España. Por otra parte, hemos agrupado a todos los adolescentes latinoamericanos en un mismo epígrafe, el de latinos, cuando existe una gran diversidad entre ellos, dejando para otro trabajo las posibles matizaciones en función de la heterogeneidad de los mismos. No obstante, consideramos que este trabajo aporta datos relevantes sobre los consumos intensivos de las drogas más accesibles a los adolescentes. Especialmente importante es señalar cómo los contextos más cercanos a los adolescentes latinoamericanos influyen en sus consumos y entre ellos están los amigos, tanto de sus zonas de origen como los autóctonos. Entender y conocer los patrones de consumo de los adolescentes en función de la etnicidad es crucial para dirigir los esfuerzos preventivos sobre todo en aquellos consumos que pueden hacerse persistentes en el transito a la vida adulta.

\section{BIBLIOGRAFÍA:}

(1) Secretaria General de Inmigración y Emigración. Anuario estadístico de Inmigración del año 2009. Ministerio de Empleo y Seguridad Social. (Consultado 5 marzo 2012) http://extranjeros.meyss.es/ es/ObservatorioPermanenteInmigracion/Anuarios/Anuario2009.html

(2) Gardner M. y Steinberg L. Peer influence on risk taking, risk preference, and risky decision making in adolescence and adulthood: an experimental study. Developmental Psychology. 2005; 41(4), $625-35$.

(3) France A. Towards a Sociological Understanding of Youth and their Risk-taking. Journal Youth Studies 2000; 3(3), 317-31.

(4) Best D, Rawaf S, Rowley J, Floyd K, Manning V y Strang J. Ethnic and gender differences in drinking and smoking among London Adolescents. Ethnicity \& Helath, 2001; 6(1), 51-57.

(5) Tyler KA, Torres R, Bersani B. Examining the changing influence of predictors on adolescent Alcohol Misuse. Journal of Child \& Adolescent Substance Abuse, 2006; 16(2), 95-114.

(6) Werch CH, Jobli EC, Moore MJ, Di Clemente CC, Dore HS. Brown CH. Do alcohol consumption patters of adolescents differ by beverage type? Journal of Child \& Adolescent Substance Abuse 2006; 15(3), 45-62.

(7) Rodham K, Hawton K, Evans E, Weatherall R. 2005. Ethnic and gender differences in drinking, smoking and drug taking among adolescents in England: a self-report school-based survey of 15 and 16 year olds. Journal of Adolescence 28:63-73.

(8) González AM y McNulty T. Gender, Acculturation and Alcohol Use among Latina/o Adolescents: A Multi-Ethnic comparison. Journal Inmigrant Minority Health, 2010; 12, 153-165.

(9) Siqueira LM y Crandall LA. Risk and Protective Factors for binge drinking among hispanic subgroups in Florida. Journal of Ethnicity in Substance Abuse 2008; 7(1), 81-92.

(10) Park S, Weaver TE, Romer D. 2009. Predictors of the transition from experimental to daily smoking among adolescents in the United States. JSPN. 14(2):102-111. 
(11) Swaim R, Wayman JC, y Chen J. Alcohol use among Mexican American and Non-Hispanic White 7th-12th-Grade Students in the Southwestern United States. Journal of Child \& Adolescent Substance Abuse 2004; 14(2),1-18.

(12) Tubergen F y Poortman AR. Adolescent Alcohol use in the Netherlands: role of ethnicity, ethnic intermarriage and ethnic school composition. Ethnicity \& Health, 2010; 15(1), 1-13.

(13) Clayton R. Transitions in Drug Use: Risk and Protective Factors, En M. Glantz y R. Pickens. Vulnerability to drug abuse. Washington: American Psychological Association. 1992.

(14) Meneses C, Romo N, Uroz J, Gil E, Markez I, Giménez S y Vega A. Adolescencia, consumo de drogas, y comportamientos de riesgo: Diferencias por sexo, etnicidad u áreas geográficas en España. Trastornos Adictivos 2009; 11(1), 51-63.

(15) Pérez Ramos M y Lucio-Gómez Maqueo E. Construcción de un modelo de riesgo en el consumo de alcohol y otras sustancias ilícitas en adolescentes estudiantes de Bachillerato. Salud y Drogas, 2010; 10(1), 79-96.

(16) Uribe JI, Verdugo JC, Zacarias X. Relación entre percepción de riesgo y consumo de drogas en estudiantes de bachillerato. Psicología y Salud, 2011; 21(1), 47-55.

(17) Delegación del Gobierno para el Plan Nacional sobre Drogas. Encuesta nacional del Observatorio Español sobre Drogas (ESTUDES, 2009), Situación y tendencias de los problemas de drogas en España. Madrid, 2009. (Consultado el 5 de febrero de 2013) http://www.pnsd.msc.es/Categoria2/ observa/pdf/oed-2009.pdf

(18) O’Malley PM, Johnston Ll D, y Bachman JG. (1998). Alcohol use among adolescents. (Consultado 5-mayo-2012) http://pubs.niaaa.nih.gov/publications/arh22-2/85-94.pdf

(19) Villarreal-González ME, Sánchez-Sosa JC, Musitu G y Varela R. El consumo de alcohol en adolescentes escolarizados: propuesta de un modelo sociocomunitario. Intervención Psicosocial 2010; 19(3), 253-264.

(20) Luengo MA, Villar P, Sobral J, Romero E, Gómez-Franguela JA. El consumo de drogas en los adolescentes inmigrantes: implicaciones para la prevención. Revista Española de Drogodependencias 2009; 34(4), 448-479.

(21) Kovacs FM, García M, Oliver-Frontera M, Gil del Real MT, López J, Mufraggi N y Palou P. La influencia de los padres sobre el consumo de alcohol y tabaco y otros hábitos en los adolescentes de Palma de Mallorca. Rev Esp Salud Pública 2008; 82: 677-689.

(22) Marina J. Muñoz-Rivas y José Luis Graña López Factores familiares de riesgo y de protección para el consumo de drogas en adolescentes Psicothema 2001. Vol. 13, no 1, pp. 87-94.

(23) Michaud PA, Blum R, y Ferron Ch. «Bet You I Will!»: risk or experimental behavior during adolescence? Pediatric Adolescent, 1998; 152(2), 224-6. 